\title{
About the Regularizing Properties of the Non-cut-off Kac Equation
}

\section{Laurent Desvillettes}

Université d'Orléans, Department de Mathématiques, BP 6759 F-45067 Orléans, France

Received: 15 February 1994/in revised form: 26 July 1994

\begin{abstract}
We prove in this work that under suitable assumptions, the solution of the spatially homogeneous non-cut-off Kac equation (or of the spatially homogeneous non cut-off 2D Boltzmann equation with Maxwellian molecules in the radial case) becomes very regular with respect to the velocity variable as soon as the time is strictly positive.
\end{abstract}

\section{Introduction}

In the upper atmosphere, a gas is described by the nonnegative density $f(t, x, v)$ of particles which at time $t$ and point $x$, move with velocity $v$. Such a density satisfies the Boltzmann equation (cf. [Ce], [Ch, Co], [Tr, Mu]):

$$
\frac{\partial f}{\partial t}+v \cdot \nabla_{x} f=Q(f)
$$

where $Q$ is a quadratic collision kernel acting only on the variable $v$ and taking in account any collisions preserving momentum and kinetic energy:

$$
\begin{aligned}
Q(f)(v)= & \int_{v_{*} \in \mathbb{R}^{3}} \int_{\theta=0}^{\pi} \int_{\phi=0}^{2 \pi}\left\{f\left(v^{\prime}\right) f\left(v_{*}^{\prime}\right)\right. \\
& \left.-f(v) f\left(v_{*}\right)\right\} B\left(\left|v-v_{*}\right|, \theta\right) \sin \theta d \phi d \theta d v_{*},
\end{aligned}
$$

with

$$
\begin{gathered}
v^{\prime}=\frac{v+v_{*}}{2}+\frac{\left|v-v_{*}\right|}{2} \sigma, \\
v_{*}^{\prime}=\frac{v+v_{*}}{2}-\frac{\left|v-v_{*}\right|}{2} \sigma, \\
\cos \theta=\sigma \cdot \frac{v-v_{*}}{\left|v-v_{*}\right|},
\end{gathered}
$$


and $B$ is a nonnegative cross section. When the collisions in the gas come out of an inverse power law interaction in $\frac{1}{r^{s}}$ (with $s \geqq 2$ ), the cross section writes

$$
B(x, \theta)=x^{\frac{s-5}{s-1}} b(\theta)
$$

where $\left.\left.b \in L_{\mathrm{loc}}^{\infty}(] 0, \pi\right]\right)$ and

$$
\sin \theta b(\theta) \sim K(s) \theta^{-\frac{s+1}{s-1}}
$$

for some $K(s)>0$ when $\theta \rightarrow 0$.

Most of the mathematical work about the Boltzmann equation is made under the assumption of angular cut-off of Grad (cf. [Gr]), which means that $b$ in Eq. (1.6) is supposed to satisfy $\sin \theta b(\theta) \in L^{1}([0, \pi])$. Note that for inverse power laws in $\frac{1}{r^{s}}$ with $s \geqq 2$, this assumption never holds (because of the singularity appearing in Eq. (1.7)).

For example, the existence of a global renormalized solution to the full Boltzmann equation (1.1) is known under this assumption (cf. [DP, L]), but it is also the case with most of the works concerning the spatially homogeneous Boltzmann equation (cf. [A 1], [A 3], [Ee], [De 1]):

$$
\frac{\partial f}{\partial t}(t, v)=Q(f)(t, v)
$$

with the noticeable exception of [A 2], where existence is proved for the non-cut-off equation (1.2)-(1.8) when $s>3$.

We shall now concentrate on this spatially homogeneous equation (1.8). When the cut-off assumption is made, it is possible to write

$$
Q(f)=Q^{+}(f)-f L f
$$

where

$$
Q^{+}(f)(v)=\int_{v_{*} \in \mathbb{R}^{3}} \int_{\theta=0}^{\pi} \int_{\phi=0}^{2 \pi} f\left(v^{\prime}\right) f\left(v_{*}^{\prime}\right) B\left(\left|v-v_{*}\right|, \theta\right) \sin \theta d \phi d \theta d v_{*},
$$

and

$$
L f=A * f,
$$

with

$$
A(x)=2 \pi \int_{\theta=0}^{\pi} B(|x|, \theta) \sin \theta d \theta .
$$

Then, the solution $f(t, v)$ of Eq. (1.8) can be written under the form

$$
f(t, v)=f(0, v) e^{-\int_{0}^{t} L f(\tau, v) d \tau}+\int_{0}^{t} Q^{+}(f)(s, v) e^{-\int_{s}^{t} L f(\tau, v) d \tau} d s .
$$

But the operator $Q^{+}$is known to be regularizing with respect to the variable $v$ (at least when $f \in L^{2}\left(\mathbb{R}_{v}^{3}\right)$, and when $B$ satisfies some properties) (cf. [L 1]). Therefore, if $f(0, v)$ is not regular (for example if it belongs to $L^{2}\left(\mathbb{R}_{v}^{3}\right)$ but not to $H^{1}\left(\mathbb{R}_{v}^{3}\right)$ ), the solution $f(t, v)$ of Eq. (1.8) will at best keep the regularity of $f(0, v)$ 
when $t>0$. In particular, no regularizing effect is expected for the solution of the cut-off homogeneous Boltzmann equation (1.8).

On the other hand, one can hope some regularizing properties for the solution of the non-cut-off homogeneous Boltzmann equation (1.8), (1.2) (when (1.6), (1.7) holds).

One of the reasons of assuming such a conjecture is that an asymptotics of the Boltzmann equation when the cross section is concentrating on the grazing collisions (these collisions are those that are neglected when the cut-off assumption is made) leads to the Fokker-Planck-Landau equation (cf. [De 2], [Dg, Lu]), which is known to induce regularizing effects (or at least compactness properties, even in the spatially inhomogeneous case (cf.[L 2])).

This article is devoted to the proof of such a conjecture in the simpler case of spatially homogeneous Kac equation. We recall that the original Kac model is used to describe a one-dimensional spatially homogeneous gas in which the collisions preserve the mass and the energy, but not the momentum (cf. $[\mathrm{K}],[\mathrm{MK}]$ ).

Note also that the theorems of Sects. 2, 3 and 4 hold for the spatially homogeneous non cut-off 2D radially symmetric solutions of the Boltzmann equation with Maxwellian molecules, as is shown in Appendix C.

In the Kac model, the nonnegative density $f(t, v)$ satisfies

$$
\frac{\partial f}{\partial t}(t, v)=K(f)(t, v)
$$

where

$$
K(f)(t, v)=\int_{v_{*} \in \mathbb{R}} \int_{\theta=-\pi}^{\pi}\left\{f\left(v^{\prime \prime}\right) f\left(v_{*}^{\prime \prime}\right)-f(v) f\left(v_{*}\right)\right\} \frac{d \theta}{2 \pi} d v_{*},
$$

and

$$
\begin{aligned}
& v^{\prime \prime}=v \cos \theta-v_{*} \sin \theta, \\
& v_{*}^{\prime \prime}=v \sin \theta+v_{*} \cos \theta .
\end{aligned}
$$

The analysis leading to Eq. (1.13) still holds in this case. Therefore, one can at best hope that the regularity of $f(0, v)$ is conserved for the solution $f(t, v)$ of Eq. (1.14) when $t>0$. This affirmation is indeed easily proved when $f(0, v) \in$ $L^{1}\left(\left(1+|v|^{2}\right) d v\right)$ (cf. Theorem A.1 of Appendix A), but also in the more difficult case when $f(0, v)$ lies in some Hölder spaces (cf. [G]) (note also the results in the same spirit for the Boltzmann equation of [We]).

We will therefore concentrate in this work on the equation

$$
\frac{\partial f}{\partial t}(t, v)=K_{\beta}(f)(t, v)
$$

where

$$
K_{\beta}(f)(t, v)=\int_{v_{*} \in \mathbb{R}} \int_{0=-\pi}^{\pi}\left\{f\left(v^{\prime \prime}\right) f\left(v_{*}^{\prime \prime}\right)-f(v) f\left(v_{*}\right)\right\} \beta(|\theta|) d \theta d v_{*},
$$

with

$$
\beta(x) \sim x^{-x}
$$

When $x \rightarrow 0^{+}$and $\left.\left.\alpha \in\right] 1,3\right]$.

This kernel is obtained by analogy with the non cut-off kernel (1.2), (1.6), (1.7) of Boltzmann equation. 
However, the analysis in the case when $\alpha=3$ (corresponding to the Coulombian interaction in the case of the Boltzmann equation (cf. [Dg, Lu])) is very different from the analysis when $\alpha \in] 1,3[$. Therefore, we will only consider in the sequel the latter case.

We begin in Sect. 2 by proving that the existence of a solution holds for Eq. $(1.18)-(1.20)$. We prove then in Sect. 3 our main theorem. Namely, if $f(0, v) \in$ $L^{1}\left(\mathbb{R}_{v},\left(1+|v|^{\gamma}\right) d v\right)$ for all $\gamma>0$, the solution $f(t, v)$ of Eq. (1.18)-(1.20) lies in $C^{\infty}\left(\mathbb{R}_{v}\right)$ for all $t>0$. Finally, in Sect. 4 , we consider the case when only a finite number of moments are known to be initially bounded for $f$. The reader will also find for the sake of completeness some classical results used throughout this work in Appendix A and B at the end of the paper, Appendix $\mathrm{C}$ being devoted to the extension of the results to the $2 \mathrm{D}$ radially symmetric Boltzmann equation with Maxwellian molecules.

\section{Existence for the Non cut-off Kac Equation}

We prove in this section the following theorem:

Theorem 2.1. Let $f_{0} \geqq 0$ be an initial datum such that

$$
\int_{v \in \mathbb{R}} f_{0}(v)\left(1+|v|^{2}+\left|\log f_{0}(v)\right|\right) d v<+\infty,
$$

and let $\beta \geqq 0$ be a cross section satisfying the following property:

$$
\left.\left.\exists \beta_{0}, \beta_{1}>0, \alpha \in\right] 1,3[, \quad \forall x \in] 0, \pi\right], \quad \beta_{0}|x|^{-\alpha} \leqq \beta(x) \leqq \beta_{1}|x|^{-\alpha} .
$$

Then, there exists a nonnegative solution $f(t, v) \in L^{\infty}\left(\left[0,+\infty\left[t ; L^{1}\left(\mathbb{R}_{v},(1+\right.\right.\right.\right.$ $\left.\left.|v|^{2}\right) d v\right)$ ) to Eq. (1.18), (1.19), (2.2) with initial datum $f_{0}$ in the following sense:

For all functions $\phi \in W^{2, \infty}\left(\mathbb{R}_{v}\right)$, we have

$$
\frac{\partial}{\partial t} \int_{v \in \mathbb{R}} f(t, v) \phi(v) d v=\int_{v \in \mathbb{R}} \int_{v_{*} \in \mathbb{R}} K^{\phi}\left(v, v_{*}\right) f(t, v) f\left(t, v_{*}\right) d v_{*} d v,
$$

where

$$
K^{\phi}\left(v, v_{*}\right)=\int_{\theta=-\pi}^{\pi}\left\{\phi\left(v^{\prime \prime}\right)-\phi(v)\right\} \beta(|\theta|) d \theta .
$$

The conservation of mass

$$
\int_{v \in \mathbb{R}} f(t, v) d v=\int_{v \in \mathbb{R}} f_{0}(v) d v
$$

holds for these solutions, but the energy may decrease.

Moreover, if for some $p \in \mathbb{N}$, there exists $C_{2.1}>0$ such that

$$
\int_{v \in \mathbb{R}} f_{0}(v)\left(1+|v|^{2 p}\right) d v \leqq C_{2.1}
$$

one can find $C_{2.2}>0$ such that for all $t \geqq 0$,

$$
\int_{v \in \mathbb{R}} f(t, v)\left(1+|v|^{2 p}\right) d v \leqq C_{2.2} .
$$


Finally, if assumption (2.6) holds for some $p \geqq 2$, the conservation of energy

$$
\int_{v \in \mathbb{R}} f(t, v)|v|^{2} d v=\int_{v \in \mathbb{R}} f_{0}(v)|v|^{2} d v
$$

holds.

Remark. The analogue of this theorem is proved in [A 2] for the Boltzmann equation (with $s>3$ ). The proof given is very similar to that of [A 2].

The sense to give to the right term of Definition (2.4) will become clear in the sequel. Note however that because of the singularity of $\beta$, this term is not defined if $\phi$ is not regular $\left(W^{2, \infty}\right)$.

Proof of Theorem 2.1. We introduce for all $n \in \mathbb{N}^{*}$ the truncated sequence

$$
\beta_{n}=\beta \wedge n \text {. }
$$

Note that because of assumption (2.2), there exists for all $\gamma>\alpha-1$ a strictly positive $C_{2.3}(\gamma)$ such that for all $n \in \mathbb{N}^{*}$,

$$
\int_{0=-\pi}^{\pi}\left(|1-\cos \theta|^{\gamma / 2}+|\sin \theta|^{\gamma}\right) \beta_{n}(|\theta|) d \theta \leqq C_{2.3}(\gamma)
$$

It is also clear that

$$
\int_{\theta=-\pi}^{\pi}\left(|1-\cos \theta|^{\gamma / 2}+|\sin \theta|^{\eta}\right)\left|\beta(|\theta|)-\beta_{n}(|\theta|)\right| d \theta \underset{n \rightarrow+\infty}{\longrightarrow} 0 .
$$

Then, we consider the (unique) nonnegative solution $f_{n}(t, v)$ of the classical Kac equation

$$
\frac{\partial f_{n}}{\partial t}(t, v)=K_{\beta_{n}}\left(f_{n}\right)(t, v)
$$

with initial datum $f_{0}$ (for the existence and uniqueness of such a solution, cf. Theorem A.1 of Appendix A). This solution is known to satisfy the conservation of mass and energy, and the entropy inequality (cf. Theorem A.1 and A.2 of Appendix A):

$$
\begin{aligned}
\int_{v \in \mathbb{R}} f_{n}(t, v) d v & =\int_{v \in \mathbb{R}} f_{0}(v) d v, \\
\int_{v \in \mathbb{R}} f_{n}(t, v)|v|^{2} d v & =\int_{v \in \mathbb{R}} f_{0}(v)|v|^{2} d v, \\
\int_{v \in \mathbb{R}} f_{n}(t, v) \log f_{n}(t, v) d v & \leqq \int_{v \in \mathbb{R}} f_{0}(v) \log f_{0}(v) d v .
\end{aligned}
$$

It is now classical (cf. [De 3] for example) that Eq. (2.13) - (2.15) ensure the existence of a constant $C_{2.4}$ such that

$$
\int_{v \in \mathbb{R}} f_{n}(t, v)\left(1+|v|^{2}+\left|\log f_{n}(t, v)\right|\right) d v \leqq C_{2.4} .
$$

Because of the Dunford-Pettis theorem (cf. [B]) and of estimate (2.16), one can extract from $\left(f_{n}\right)_{n \in \mathbb{N}}$ a subsequence still denoted by $\left(f_{n}\right)_{n \in \mathbb{N}}$ and converging to a function $f$ in $L^{\infty}\left(\left[0,+\infty\left[t ; L^{1}\left(\mathbb{R}_{v}\right)\right)\right.\right.$ weak ${ }^{*}$.

Moreover, for all $q \in L^{1}\left(\left[0,+\infty[)\right.\right.$ and all $\psi \in L_{\mathrm{loc}}^{\infty}\left(\left[0,+\infty\left[{ }_{t} \times \mathbb{R}_{v}\right)\right.\right.$ such that

$$
\lim _{|v| \rightarrow+\infty} \sup _{t \in \mathbb{R}} \frac{|\psi(t, v)|}{|v|^{2}}=0,
$$


we have

$$
\int_{0}^{+\infty} q(t) \int_{v \in \mathbb{R}} f_{n}(t, v) \psi(t, v) d v d t \underset{n \rightarrow+\infty}{\longrightarrow} \int_{0}^{+\infty} q(t) \int_{v \in \mathbb{R}} f(t, v) \psi(t, v) d v d t
$$

Denoting for all $\phi \in W^{2, \infty}\left(\mathbb{R}_{v}\right)$,

$$
K_{n}^{\phi}\left(v, v_{*}\right)=\int_{\theta=-\pi}^{\pi}\left\{\phi\left(v^{\prime \prime}\right)-\phi(v)\right\} \beta_{n}(|\theta|) d \theta
$$

it is clear that (using the change of variables $\left(v, v_{*}, \theta\right) \rightarrow\left(v^{\prime \prime}, v_{*}^{\prime \prime},-\theta\right)$ ),

$$
\frac{\partial}{\partial t} \int_{v \in \mathbb{R}} f_{n}(t, v) \phi(v) d v=\int_{v \in \mathbb{R}} \int_{v_{*} \in \mathbb{R}} K_{n}^{\phi}\left(v, v_{*}\right) f_{n}(t, v) f_{n}\left(t, v_{*}\right) d v_{*} d v .
$$

We shall now prove that when $\phi \in W^{2, \infty}\left(\mathbb{R}_{v}\right)$, it is possible to pass to the limit in Eq. (2.20) and to obtain Eq. (2.3). We begin by the

Lemma 1. There exists a constant $C_{2.5}>0$ (depending on $\alpha$ ) and a sequence $C_{2.5}(n)$ converging to 0 such that the following estimates hold:

1. for all $\phi \in W^{2, \infty}\left(\mathbb{R}_{v}\right)$,

$$
\left|K_{n}^{\phi}\left(v, v_{*}\right)\right| \leqq C_{2.5}\left(1+|v|^{\frac{\alpha+5}{4}}+\left|v_{*}\right|^{\frac{\alpha+5}{4}}\right)\|\phi\|_{W^{2, \infty}\left(\mathbb{R}_{v}\right)},
$$

2. for all $\phi \in W^{2, \infty}\left(\mathbb{R}_{v}\right)$,

$$
\left|K^{\phi}\left(v, v_{*}\right)-K_{n}^{\phi}\left(v, v_{*}\right)\right| \leqq C_{2.5}(n)\left(1+|v|^{\frac{\alpha+5}{4}}+\left|v_{*}\right|^{\frac{\alpha+5}{4}}\right)\|\phi\|_{W^{2, \infty}\left(\mathbb{R}_{v}\right)} .
$$

Proof of Lemma 1. Note that

$$
\begin{aligned}
\phi\left(v^{\prime \prime}\right)-\phi(v)= & \phi\left(v \cos \theta-v_{*} \sin \theta\right)-\phi(v) \\
= & \left(v(\cos \theta-1)-v_{*} \sin \theta\right) \phi^{\prime}(v)+\left(v(\cos \theta-1)-v_{*} \sin \theta\right)^{2} \\
& \times \int_{u=0}^{1}(1-u) \phi^{\prime \prime}\left(v+u\left(v(\cos \theta-1)-v_{*} \sin \theta\right)\right) d u
\end{aligned}
$$

Therefore, for all $\delta \in] 0,1[$,

$$
\begin{aligned}
&\left|\phi\left(v^{\prime \prime}\right)-\phi(v)+v_{*} \sin \theta \phi^{\prime}(v)\right| \leqq\left|\phi\left(v^{\prime \prime}\right)-\phi(v)+v_{*} \sin \theta \phi^{\prime}(v)\right|^{1-\delta} \\
& \quad \times \mid v(\cos \theta-1) \phi^{\prime}(v)+\left(v(\cos \theta-1)-v_{*} \sin \theta\right)^{2} \\
& \quad \times\left.\int_{u=0}^{1}(1-u) \phi^{\prime \prime}\left(v+u\left(v(\cos \theta-1)-v_{*} \sin \theta\right)\right) d u\right|^{\delta} \\
& \leqq 8\left(1+\left|v_{*}\right|^{1-\delta}\right)\left(3\|\phi\|_{W^{1, \infty}\left(\mathbb{R}_{v}\right)}\right)^{1-\delta}\left(|\cos \theta-1|^{\delta}+|\sin \theta|^{2 \delta}\right) \\
& \times\left(1+|v|^{2 \delta}+\left|v_{*}\right|^{2 \delta}\right)\|\phi\|_{W^{2, \infty}\left(\mathbb{R}_{v}\right)}^{\delta} \\
& \leqq C_{2.6}\|\phi\|_{W^{2, \infty}\left(\mathbb{R}_{v}\right)}\left(|\cos \theta-1|^{\delta}+|\sin \theta|^{2 \delta}\right)\left(1+|v|^{1+\delta}+\left|v_{*}\right|^{1+\delta}\right)
\end{aligned}
$$


for some strictly positive constant $C_{2.6}$. But $\theta \rightarrow \sin \theta$ is odd and therefore

$$
\begin{aligned}
\left|K_{n}^{\phi}\left(v, v_{*}\right)\right|= & \int_{\theta=-\pi}^{\pi}\left\{\phi\left(v^{\prime \prime}\right)-\phi(v)\right\} \beta_{n}(|\theta|) d \theta \mid \\
= & \left|\int_{\theta=-\pi}^{\pi}\left\{\phi\left(v^{\prime \prime}\right)-\phi(v)+v_{*} \sin \theta \phi^{\prime}(v)\right\} \beta_{n}(|\theta|) d \theta\right| \\
\leqq & C_{2.6}\|\phi\|_{W^{2, \infty}\left(\mathbb{R}_{v}\right)} \int_{\theta=-\pi}^{\pi}\left(|\cos \theta-1|^{\delta}+|\sin \theta|^{2 \delta}\right) \beta_{n}(|\theta|) d \theta \\
& \times\left(1+|v|^{1+\delta}+\left|v_{*}\right|^{1+\delta}\right) .
\end{aligned}
$$

We now use Eq. (2.10) with $\delta=\frac{1+\alpha}{4}$, and obtain

$$
\left|K_{n}^{\phi}\left(v \cdot v_{*}\right)\right| \leqq 2 C_{2.3}\left(\frac{1+\alpha}{2}\right) C_{2.6}\left(1+|v|^{\frac{\alpha+5}{4}}+\left|v_{*}\right|^{\frac{\alpha+5}{4}}\right)\|\phi\|_{W^{2, \infty}\left(\mathbb{R}_{v}\right)}
$$

which clearly implies estimate (2.21). In order to get estimate (2.22), we use exactly the same proof, except that Eq. (2.10) is replaced by Eq. (2.11).

Lemma 2. There exists a constant $C_{2.7}>0$ (depending on $\alpha$ ) such that when $\phi \in W^{2, \infty}\left(\mathbb{R}_{v}\right)$ satisfies

$$
\||\phi|\|=\sup _{v \in \mathbb{R}} \frac{\left|\phi^{\prime}(v)\right|}{1+|v|}<+\infty,
$$

one has the following estimate:

$$
\left|K_{n}^{\phi}\left(v, v_{*}\right)\right| \leqq C_{2.7}\left(\left\|\phi^{\prime \prime}\right\|_{L^{\infty}\left(\mathbb{R}_{v}\right)}+|\| \phi|||\right)\left(1+|v|^{2}+\left|v_{*}\right|^{2}\right)
$$

Proof of Lemma 2. According to Eq. (2.23),

$$
\begin{aligned}
& \left|\phi\left(v^{\prime \prime}\right)-\phi(v)+v_{*} \sin \theta \phi^{\prime}(v)\right|=\mid v(\cos \theta-1) \phi^{\prime}(v) \\
& \quad+\left(v(\cos \theta-1)-v_{*} \sin \theta\right)^{2} \int_{u=0}^{1}(1-u) \phi^{\prime \prime}\left(v+u\left(v(\cos \theta-1)-v_{*} \sin \theta\right)\right) d u \mid \\
& \quad \leqq|\cos \theta-1||v|\left|\phi^{\prime}(v)\right|+4\left(|\cos \theta-1|+|\sin \theta|^{2}\right)\left(|v|^{2}+\left|v_{*}\right|^{2}\right)\left\|\phi^{\prime \prime}\right\|_{L^{\infty}\left(\mathbb{R}_{v}\right)} \\
& \leqq C_{2.8}\left(|\cos \theta-1|+|\sin \theta|^{2}\right)\left(1+|v|^{2}+\left|v_{*}\right|^{2}\right)\left(\left\|\phi^{\prime \prime}\right\|_{L^{\infty}\left(\mathbb{R}_{v}\right)}+\|\phi|\||)\right.
\end{aligned}
$$

for some constant $C_{2.8}>0$.

Using now the oddity of $\theta \rightarrow \sin \theta$ as in Lemma 1, and estimate (2.10) for $\gamma=2$, we get estimate $(2.28)$. 
We now come back to the proof of Theorem 2.1.

Suppose that $\phi \in W^{2, \infty}\left(\mathbb{R}_{v}\right), q \in L^{1}([0,+\infty[)$, and $v \in \mathbb{R}$. Then, because of Lemma 1,

$$
\begin{aligned}
&\left|\int_{t=0}^{+\infty} \int_{v_{*} \in \mathbb{R}} K_{n}^{\phi}\left(v, v_{*}\right) f_{n}\left(t, v_{*}\right) d v_{*} q(t) d t-\int_{t=0}^{+\infty} \int_{v_{*} \in \mathbb{R}} K^{\phi}\left(v, v_{*}\right) f\left(t, v_{*}\right) d v_{*} q(t) d t\right| \\
& \leqq \int_{t=0}^{+\infty} \int_{v_{*} \in \mathbb{R}}\left|K_{n}^{\phi}\left(v, v_{*}\right)-K^{\phi}\left(v, v_{*}\right)\right| f_{n}\left(t, v_{*}\right) d v_{*} q(t) d t \\
&+\left|\int_{t=0}^{+\infty} \int_{v_{*} \in \mathbb{R}} K^{\phi}\left(v, v_{*}\right)\left\{f_{n}\left(t, v_{*}\right)-f\left(t, v_{*}\right)\right\} d v_{*} q(t) d t\right| \\
& \leqq C_{2.5}(n)\|q\|_{L^{1}([0,+\infty[t)}|| \phi \|_{W^{2, \infty}\left(\mathbb{R}_{v}\right)} \int_{v_{*} \in \mathbb{R}}\left(1+|v|^{\frac{\alpha+5}{4}}+\left|v_{*}\right|^{\frac{\alpha+5}{4}}\right) f_{n}\left(t, v_{*}\right) d v_{*} \\
&+\left|\int_{t=0}^{+\infty} \int_{v_{*} \in \mathbb{R}} K^{\phi}\left(v, v_{*}\right)\left\{f_{n}\left(t, v_{*}\right)-f\left(t, v_{*}\right)\right\} d v_{*} q(t) d t\right|
\end{aligned}
$$

But the first term of Eq. (2.30) tends to 0 because of estimate (2.16). Moreover, because of Lemma 1 , we have for all $v \in \mathbb{R}$,

$$
\lim _{\left|v_{*}\right| \rightarrow+\infty} \frac{\left|K^{\phi}\left(v, v_{*}\right)\right|}{\left|v_{*}\right|^{2}}=0 .
$$

Therefore, estimate (2.18) ensures that the second term of Eq. (2.30) tends to 0 . Finally, we obtain for all $\phi \in W^{2, \infty}\left(\mathbb{R}_{v}\right)$ and $v \in \mathbb{R}$, the convergence in $L^{\infty}\left(\left[0,+\infty[t)\right.\right.$ weak ${ }^{*}$ of

$$
L_{n}^{\phi}(t, v)=\int_{v_{*} \in \mathbb{R}} K_{n}^{\phi}\left(v, v_{*}\right) f_{n}\left(t, v_{*}\right) d v_{*}
$$

towards

$$
L^{\phi}(t, v)=\int_{v_{*} \in \mathbb{R}} K^{\phi}\left(v, v_{*}\right) f\left(t, v_{*}\right) d v_{*} .
$$

We now observe that for all $\phi \in W^{2, \infty}\left(\mathbb{R}_{v}\right)$ and $v \in \mathbb{R}$, the sequence

$$
\Theta_{n}^{\phi}\left(v, v_{*}\right)=\frac{\partial^{2} K_{n}^{\phi}}{\partial v_{*}^{2}}\left(v, v_{*}\right)
$$

is bounded in $L^{\infty}\left(\mathbb{R}_{v}\right)$. More precisely,

$$
\begin{aligned}
\left|\frac{\partial^{2} K_{n}^{\phi}}{\partial v_{*}^{2}}\left(v, v_{*}\right)\right| & =\left|\int_{\theta=-\pi}^{\pi} \sin ^{2} \theta \phi^{\prime \prime}\left(v^{\prime \prime}\right) \beta_{n}(|\theta|) d \theta\right| \\
& \leqq C_{2.3}(2)\left\|\phi^{\prime \prime}\right\|_{L^{\infty}\left(\mathbb{R}_{v}\right)} .
\end{aligned}
$$

Moreover,

$$
\begin{aligned}
\left|\frac{\partial K_{n}^{\phi}}{\partial v_{*}}\left(v, v_{*}\right)\right| & =\left|\int_{\theta=-\pi}^{\pi} \sin \theta \phi^{\prime}\left(v^{\prime \prime}\right) \beta_{n}(|\theta|) d \theta\right| \\
& =\left|\int_{\theta=-\pi}^{\pi}-\sin \theta\left\{\phi^{\prime}\left(v^{\prime \prime}\right)-\phi^{\prime}(v)\right\} \beta_{n}(|\theta|) d \theta\right|
\end{aligned}
$$




$$
\begin{aligned}
& \leqq \\
& \qquad \int_{\theta=-\pi}^{\pi}|\sin \theta|\left\{|v||\cos \theta-1|+\left|v_{*}\right||\sin \theta|\right\} \\
& \quad \times \int_{u=0}^{1}\left|\phi^{\prime \prime}\left(v+u\left(v(\cos \theta-1)-v_{*} \sin \theta\right)\right)\right| d u \beta_{n}(|\theta|) d \theta \\
& \leqq 2 C_{2.3}(2)\left\|\phi^{\prime \prime}\right\|_{L^{\infty}}\left(1+|v|+\left|v_{*}\right|\right)
\end{aligned}
$$

because of estimate (1.10).

Therefore, using Lemma 2 , for all $\phi \in W^{2, \infty}\left(\mathbb{R}_{v}\right)$ and $v \in \mathbb{R}$,

$$
\begin{aligned}
& \left|\frac{\partial L_{n}^{\phi}}{\partial t}(t, v)\right|=\left|\frac{\partial}{\partial t} \int_{v_{*} \in \mathbb{R}} K_{n}^{\phi}\left(v, v_{*}\right) f_{n}\left(t, v_{*}\right) d v_{*}\right| \\
& =\left|\int_{w \in \mathbb{R}} \int_{v_{*} \in \mathbb{R}} K_{n}^{K_{n}^{\phi}(v, \cdot)}\left(w, v_{*}\right) f_{n}(t, w) f_{n}\left(t, v_{*}\right) d v_{*} d w\right| \\
& \leqq \int_{w \in \mathbb{R}} \int_{v_{*} \in \mathbb{R}} C_{2.7}\left(\left\|\frac{\partial^{2} K_{n}^{\phi}}{\partial v_{*}^{2}}(v, \cdot)\right\|_{L^{\infty}\left(\mathbb{R}_{v_{*}}\right)}+\left\|\mid K_{n}^{\phi}(v, \cdot)\right\| \|\right) \\
& \times\left(1+|w|^{2}+\left|v_{*}\right|^{2}\right) f_{n}(t, w) f_{n}\left(t, v_{*}\right) d v_{*} d w \\
& \leqq C_{2.4}^{2} C_{2.7}\left\{C_{2.3}(2)\left\|\phi^{\prime \prime}\right\|_{L^{\infty}\left(\mathbb{R}_{\iota}\right)}+2 C_{2.3}(2) C_{2.5}(1+|v|)\left\|\phi^{\prime \prime}\right\|_{L^{\infty}\left(\mathbb{R}_{\iota}\right)}\right\} \text {. }
\end{aligned}
$$

It is also clear that

$$
\begin{aligned}
\left|L_{n}^{\phi}(t, v)\right| & \leqq \int_{v * \in \mathbb{R}} C_{2.5}\left(1+|v|^{\frac{\alpha+5}{4}}+\left|v_{*}\right|^{\frac{\alpha+5}{4}}\right) f_{n}\left(t, v_{*}\right) d v_{*} \mid\|\phi\|_{W^{2, \infty}\left(\mathbb{R}_{v}\right)} \\
& \leqq C_{2.4} C_{2.5}\left(1+|v|^{\frac{\alpha+5}{4}}\right)\|\phi\|_{W^{2, \infty}\left(\mathbb{R}_{v}\right)}
\end{aligned}
$$

Therefore, for all $\phi \in W^{2, \infty}\left(\mathbb{R}_{v}\right)$ and $v \in \mathbb{R}$, the sequence $L_{n}^{\phi}(\cdot, v)$ is bounded in $W^{1, \infty}([0,+\infty[t)$. Using now the weak convergence $(2.33)$ and Rellich theorem (cf. [B]), it is clear that for all $\phi \in W^{2, \infty}\left(\mathbb{R}_{v}\right)$, and a.e. $(t, v) \in\left[0,+\infty\left[{ }_{t} \times \mathbb{R}_{v}\right.\right.$, the sequence $L_{n}^{\phi}$ tends to $L^{\phi}$. Therefore, for all $q \in L^{1}([0,+\infty[)$ and all $T>0$ such that Supp $q \subset[0, T]$,

$$
\begin{aligned}
& \mid \int_{t=0}^{+\infty}\left\{\int_{v \in \mathbb{R}} \int_{v_{*} \in \mathbb{R}} K_{n}^{\phi}\left(v, v_{*}\right) f_{n}(t, v) f_{n}\left(t, v_{*}\right) d v d v_{*}\right. \\
& \left.\quad-\int_{v \in \mathbb{R}} \int_{v_{*} \in \mathbb{R}} K^{\phi}\left(v, v_{*}\right) f(t, v) f\left(t, v_{*}\right) d v d v_{*}\right\} q(t) d t \mid \\
& =\left|\int_{t=0}^{+\infty}\left\{\int_{v \in \mathbb{R}} L_{n}^{\phi}(t, v) f_{n}(t, v) d v-\int_{v \in \mathbb{R}} L^{\phi}(t, v) f(t, v) d v\right\} q(t) d t\right| \\
& \leqq \\
& \quad \sup _{t \in[0, T]}\left\{\int_{v \in \mathbb{R}}\left|L_{n}^{\phi}(t, v)-L^{\phi}(t, v)\right| f_{n}(t, v) d v\right\}\|q\|_{L^{1}([0,+\infty[t)} \\
& \quad+\left|\int_{t=0}^{+\infty}\left\{\int_{v \in \mathbb{R}} L^{\phi}(t, v)\left(f_{n}(t, v)-f(t, v)\right) d v\right\} q(t) d t\right| .
\end{aligned}
$$


But according to estimate (2.38),

$$
\lim _{\left|v_{*}\right| \rightarrow+\infty} \sup _{t \in[0,+\infty[} \sup _{n \in \mathbb{N}^{*}} \frac{\left|L_{n}^{\phi}\left(t, v_{*}\right)\right|}{\left|v_{*}\right|^{2}}=0 .
$$

Therefore, estimate (2.18) ensures that the second term of (2.39) tends to 0.

We finally use Egorov's theorem, estimate (2.38), the equiintegrability of the sequence $f_{n}$ (obtained by estimate $(2.16)$ and the convergence a.e. of $L_{n}^{\phi}$ to $L^{\phi}$, in order to obtain the convergence of the first term of (2.39) to 0 .

As announced before, we can now pass to the limit in Eq. (2.20) and obtain the first part of Theorem 2.1.

In order to prove the second part of Theorem 2.1, we observe that if assumption (2.6) holds, then Theorem A.2 (cf. Appendix A) ensures the existence of $C_{A .3}>0$ such that

$$
\int_{v \in \mathbb{R}} f_{n}(t, v)\left(1+|v|^{2 p}\right) d v \leqq C_{A .3}
$$

(note that $C_{A .3}$ does not depend on $n$ ).

But estimates (2.18), (2.20) and (2.21) imply for a.e. $t \geqq 0$ the convergence of $\int_{v \in \mathbb{R}} f_{n}(t, v) \chi(v) d v$ to $\int_{v \in \mathbb{R}} f(t, v) \chi(v) d v$ when $\chi \in C_{c}^{2}\left(\mathbb{R}_{v}\right)$.

Therefore, for all $R>0, t>0$,

$$
\int_{|v| \leqq R} f(t, v)\left(1+|v|^{2 p}\right) d v \leqq C_{A .3} .
$$

Then, estimate (2.7) holds because of Fatou's lemma.

Finally, we prove the conservation of mass (2.5). We observe that for some function $\chi_{R} \in C^{2}\left(\mathbb{R}_{v}\right)$ such that $\operatorname{Supp}\left(\chi_{R}\right) \subset[-R-1, R+1]$,

$$
\begin{aligned}
\left|\int_{v \in \mathbb{R}} f(t, v) d v-\int_{v \in \mathbb{R}} f_{0}(v) d v\right| \leqq & \frac{1}{R^{2}} \int_{|v| \geqq R}\left\{f_{n}(t, v)+f(t, v)\right\}|v|^{2} d v \\
& +\left|\int_{v \in \mathbb{R}} \chi_{R}(v)\left\{f_{n}(t, v)-f(t, v)\right\} d v\right|
\end{aligned}
$$

for any $R>0$. But according to the properties used in the proof of estimate (2.42), estimate (2.43) ensures that the conservation of mass (2.5) holds.

In the same way, we can see that under assumption (2.6) with $p \geqq 2$, the conservation of energy (2.8) holds.

\section{Regularization Properties When All Polynomial Moments are Initially Bounded}

This section is devoted to the proof of the following theorem:

Theorem 3.1. Let $f_{0} \geqq 0$ be an initial datum such that for all $p \in \mathbb{N}$, there exists $C_{3.1}(p)>0$ satisfying

$$
\int_{v \in \mathbb{R}} f_{0}(v)\left(1+|v|^{p}+\left|\log f_{0}(v)\right|\right) d v \leqq C_{3.1}(p),
$$

and let $\beta \geqq 0$ be a cross section satisfying estimate (2.2). 
Then, if $f(t, v)$ is a nonnegative solution of Eq. (1.18), (1.19), (2.2) in the sense of Eq. (2.3) with initial datum $f_{0}$, we have for all $\bar{t}>0$ and all $q \in \mathbb{N}$ :

$$
f(t, v) \in L^{\infty}\left(\left[\bar{t},+\infty\left[t ; C^{q}\left(\mathbb{R}_{v}\right)\right),\right.\right.
$$

or in abridged form,

$$
f(t, v) \in L^{\infty}(] 0,+\infty\left[{ }_{t} ; C^{\infty}\left(\mathbb{R}_{v}\right)\right) .
$$

Proof of Theorem 3.1. According to Theorem 2.1, we know that for all $p \in \mathbb{N}$, there exists $C_{3.2}(p)>0$ satisfying

$$
\forall t \in\left[0,+\infty\left[, \quad \int_{v \in \mathbb{R}} f(t, v)\left(1+|v|^{p}\right) d v \leqq C_{3.2}(p) .\right.\right.
$$

Therefore, the Fourier transform

$$
\hat{f}(t, \xi)=\int_{v \in \mathbb{R}} e^{-i v \xi} f(t, v) d v
$$

of $f$ is such that for all $p \in \mathbb{N}$,

$$
\left|\frac{\partial^{p} \hat{f}}{\partial \xi^{p}}(t, \xi)\right| \leqq C_{3.2}(p)
$$

But $v \rightarrow e^{-l v \xi}$ lies in $W^{2, \infty}\left(\mathbb{R}_{v}\right)$, and therefore it is possible to use Eq. (2.3).

Then, a simple calculation leads to the following equation for the Fourier transform of $f$ :

$$
\frac{\partial \hat{f}}{\partial t}(t, \xi)=\int_{\theta=-\pi}^{\pi}\{\hat{f}(t, \xi \cos \theta) \hat{f}(t, \xi \sin \theta)-\hat{f}(t, 0) \hat{f}(t, \xi)\} \beta(|\theta|) d \theta .
$$

Note that this equation is used in [G], and that it also appeared in [De 1], though for the Laplace transform of $f$. We rewrite it under the form

$$
\begin{aligned}
\frac{\partial \hat{f}}{\partial t}(t, \xi)= & \frac{1}{2} \int_{\theta=-\pi}^{\pi}\{\hat{f}(t, \xi \sin \theta)+\hat{f}(t,-\xi \sin \theta)-2 \hat{f}(t, 0)\} \beta(|\theta|) d \theta \hat{f}(t, \xi) \\
& +\int_{\theta=-\pi}^{\pi}\{\hat{f}(t, \xi \cos \theta)-\hat{f}(t, \xi)\} \hat{f}(t, \xi \sin \theta) \beta(|\theta|) d \theta
\end{aligned}
$$

We now use the notations

$$
a(t, \xi)=-\frac{1}{2} \int_{\theta=-\pi}^{\pi}\{\hat{f}(t, \xi \sin \theta)+\hat{f}(t,-\xi \sin \theta)-2 \hat{f}(t, 0)\} \beta(|\theta|) d \theta,
$$

and

$$
b(t, \xi)=\int_{\theta=-\pi}^{\pi}\{\hat{f}(t, \xi \cos \theta)-\hat{f}(t, \xi)\} \hat{f}(t, \xi \sin \theta) \beta(|\theta|) d \theta .
$$

Therefore,

$$
\frac{\partial \hat{f}}{\partial t}(t, \xi)=-a(t, \xi) \hat{f}(t, \xi)+b(t, \xi)
$$


and

$$
\hat{f}(t, \xi)=\hat{f}(0, \xi) e^{-\int_{0}^{t} a(\tau, \xi) d \tau}+\int_{0}^{t} b(s, \xi) e^{-\int_{s}^{t} a(\tau, \xi) d \tau} d s
$$

But

$$
\hat{f}(t, \xi \sin \theta)+\hat{f}(t,-\xi \sin \theta)-2 \hat{f}(t, 0) \leqq 0,
$$

because $f \geqq 0$. Therefore, $a(t, \xi)$ is real and

$$
a(t, \xi) \geqq \frac{1}{2} \int_{\theta=-\frac{\pi}{2}}^{\frac{\pi}{2}}\{2 \hat{f}(t, 0)-\hat{f}(t, \xi \sin \theta)-\hat{f}(t,-\xi \sin \theta)\} \beta(|\theta|) d \theta .
$$

Then, we make the change of variables

$$
u=|\xi| \sin \theta .
$$

We get

$$
a(t, \xi) \geqq \frac{1}{2} \int_{u=-|\xi|}^{|\xi|}\{2 \hat{f}(t, 0)-\hat{f}(t, u)-\hat{f}(t,-u)\} \beta\left(\arcsin \left|\frac{u}{\xi}\right|\right) \frac{d u}{|\xi| \sqrt{1-\left|\frac{u}{\xi}\right|^{2}}} .
$$

But for any $x \in[0, \pi]$,

$$
\beta(x) \geqq \beta_{0}|x|^{-\alpha},
$$

and therefore

$$
\begin{aligned}
a(t, \xi) & \geqq \frac{\beta_{0}}{4} \int_{u=-|\xi|}^{|\xi|}\{2 \hat{f}(t, 0)-\hat{f}(t, u)-\hat{f}(t,-u)\}\left|\frac{u}{\xi}\right|^{-\alpha} \frac{d u}{|\xi|} \\
& \geqq \frac{\beta_{0}}{4}|\xi|^{\alpha-1} \int_{u=-|\xi|}^{|\xi|}\{2 \hat{f}(t, 0)-\hat{f}(t, u)-\hat{f}(t,-u)\}|u|^{-\alpha} d u .
\end{aligned}
$$

And since

$$
\begin{aligned}
2 \hat{f}(t, 0)-\hat{f}(t, u)-\hat{f}(t,-u) & =-|u|^{2} \int_{r=-1}^{1}(1-|r|) \frac{\partial^{2} \hat{f}}{\partial \xi^{2}}(t, r u) d r \\
& =-|u|^{2} \int_{r=-1}^{1}(1-|r|) \mathscr{R} e\left(\frac{\partial^{2} \hat{f}}{\partial \xi^{2}}(t, r u)\right) d r
\end{aligned}
$$

we get

$$
a(t, \xi) \geqq \frac{\beta_{0}}{4}|\xi|^{\alpha-1} \int_{u=-|\xi|}^{|\xi|}|u|^{2-\alpha} \int_{r=-1}^{1}(1-|r|) \mathscr{R} e\left(-\frac{\partial^{2} \hat{f}}{\partial \xi^{2}}(t, r u)\right) d r d u .
$$

But

$$
-\frac{\partial^{2} \hat{f}}{\partial \xi^{2}}(t, 0)=\int_{v \in \mathbb{R}} f(t, v)|v|^{2} d v
$$


and estimate (2.8) ensures that

$$
-\frac{\partial^{2} \hat{f}}{\partial \xi^{2}}(t, 0)=\int_{v \in \mathbb{R}} f_{0}(v)|v|^{2} d v
$$

Moreover, if we denote

$$
E=\int_{v \in \mathbb{R}} f_{0}(v)|v|^{2} d v
$$

we get (thanks to estimate (3.6)), that for any $\eta$ such that

$$
|\eta| \leqq \frac{E}{C_{3.2}(3)}
$$

the estimate

$$
\mathscr{R} e\left(-\frac{\partial^{2} \hat{f}}{\partial \xi^{2}}(t, \eta)\right) \geqq \frac{E}{2}
$$

holds. But estimates (3.20) and (3.24) ensure that

$$
\begin{aligned}
a(t, \xi) & \geqq \frac{\beta_{0}}{16} E|\xi|^{\alpha-1} \int_{u=-\inf \left(|\xi|, \frac{E}{C_{3.2}(3)}\right)}^{\inf \left(|\xi|, \frac{E}{C_{3.2^{(3)}}}\right)}|u|^{2-\alpha} d u \\
& \geqq \frac{\beta_{0}}{16(3-\alpha)} E|\xi|^{\alpha-1} 2^{3-\alpha}\left(\inf \left(|\xi|, \frac{E}{C_{3.2}(3)}\right)\right)^{3-\alpha} .
\end{aligned}
$$

Therefore, there exists $C_{3.3}>0, C_{3.4}>0$, such that when $|\xi| \geqq C_{3.3}, t \geqq 0$,

$$
a(t, \xi) \geqq C_{3.4}|\xi|^{\alpha-1} \text {. }
$$

We will now use Eq. (3.12) and estimate (3.27) to prove Theorem 3.1 by induction.

Lemma 3. We make the assumptions of Theorem 3.1. We suppose moreover that there exists $\delta \geqq 0$ such that for all $t_{1}>0, \varepsilon_{1}>0$, we can find $C_{3.5}\left(\varepsilon_{1}, t_{1}\right)>0$ satisfying

$$
\forall \xi \in \mathbb{R}, \quad \sup _{s \geqq t_{1}}|\hat{f}(s, \xi)| \leqq \frac{C_{3.5}\left(\varepsilon_{1}, t_{1}\right)}{1+|\xi|^{\delta-\varepsilon_{1}}} .
$$

Then, for all $t_{1}>0, \varepsilon_{1}>0$, we can find $C_{3.6}\left(\varepsilon_{1}, t_{1}\right)>0$ satisfying

$$
\forall \xi \in \mathbb{R}, \quad \sup _{s \geqq t_{1}}|\hat{f}(s, \xi)| \leqq \frac{C_{3.6}\left(\varepsilon_{1}, t_{1}\right)}{1+|\xi|^{\delta+\frac{\alpha-1}{2}-\varepsilon_{1}}} .
$$

Proof of Lemma 3. We fix $t_{1}>0, \varepsilon_{1}>0$. According to Eq. (3.12), for any $t \geqq t_{1}$,

$$
\begin{aligned}
\hat{f}(t, \xi)= & \hat{f}(0, \xi) e^{-\int_{0}^{t} a(\tau, \xi) d \tau} \\
& +\int_{0}^{\frac{t_{1}}{2}} b(s, \xi) e^{-\int^{t} a(\tau, \xi) d \tau} d s+\int_{\frac{t_{1}}{2}}^{t} b(s, \xi) e^{-\int^{t} a(\tau, \xi) d \tau} d s .
\end{aligned}
$$


Therefore, estimate (3.27) ensures that for any $t \geqq t_{1},|\xi| \geqq C_{3.3}$,

$$
\begin{aligned}
& |\hat{f}(t, \xi)| \leqq\left|\hat{f}(0, \xi) e^{-C_{3.4} t|\xi|^{\alpha-1}}+\sup _{s \in\left[0, \frac{t_{1}}{2}\right]} \frac{t_{1}}{2}\right| b(s, \xi) \mid e^{-C_{3.4} \frac{t_{1}}{2}|\xi|^{\alpha-1}} \\
& \quad+\sup _{s \geqq \frac{t_{1}}{2}}|b(s, \xi)| \int_{\frac{t_{1}}{2}}^{t} e^{-(t-s) C_{3.4}|\xi|^{\alpha-1}} d s .
\end{aligned}
$$

But for all $s \in[0,+\infty[, \xi \in \mathbb{R}$,

$$
\begin{aligned}
|b(s, \xi)| & =\left|\int_{\theta=-\pi}^{\pi}\{\hat{f}(s, \xi \cos \theta)-\hat{f}(s, \xi)\} \hat{f}(s, \xi \sin \theta) \beta(|\theta|) d \theta\right| \\
& =\left|\int_{\theta=-\pi}^{\pi} \xi(\cos \theta-1) \int_{u=0}^{1} \frac{\partial \hat{f}}{\partial \xi}(s, \xi+u \xi(\cos \theta-1)) d u \hat{f}(s, \xi \sin \theta) \beta(|\theta|) d \theta\right| \\
& \leqq C_{2.3}(2) C_{3.2}(0) C_{3.2}(1)|\xi| .
\end{aligned}
$$

Therefore, estimate (3.31) implies that for any $t \geqq t_{1},|\xi| \geqq C_{3.3}$,

$$
\begin{aligned}
|\hat{f}(t, \xi)| \leqq & C_{3.2}(0) e^{C_{3.4} t|\xi|^{\alpha-1}}+C_{2.3}(2) C_{3.2}(0) C_{3.2}(1) \frac{t_{1}}{2}|\xi| e^{-C_{34} \frac{t_{1}}{2}|\xi|^{\alpha-1}} \\
& +\sup _{s \geqq t_{\frac{1}{2}}} \frac{|b(s, \xi)|}{C_{3.4}|\xi|^{\alpha-1}} .
\end{aligned}
$$

According to assumption (3.28), we have for all $\varepsilon>0$,

$$
\forall \xi \in \mathbb{R}, \quad \sup _{s \geqq \frac{t_{1}}{2}}|\hat{f}(s, \xi)| \leqq \frac{C_{3.5}\left(\varepsilon, t_{\frac{1}{2}}\right)}{1+|\xi|^{\delta-\varepsilon}} .
$$

Therefore, using Corollary B.3 of Appendix B and assumption (3.1), there exists for all $\varepsilon>0$ a strictly positive constant $C_{3.7}\left(\varepsilon, t_{1}\right)$ such that

$$
\forall \xi \in \mathbb{R}, \quad \sup _{s \geqq \frac{t_{1}}{2}}|\hat{f}(s, \xi)| \leqq \frac{C_{3.7}\left(\varepsilon, t_{1}\right)}{1+|\xi|^{\delta-\varepsilon}} .
$$

We now compute (for all $|\xi| \geqq C_{3.3}, \varepsilon>0$ ),

$$
\begin{aligned}
& \sup _{s \geqq \frac{t_{1}}{2}}|b(s, \xi)|=\sup _{s \geqq \frac{t_{1}}{2}}\left|\int_{\theta=-\pi}^{\pi}\{\hat{f}(s, \xi \cos \theta)-\hat{f}(s, \xi)\} \hat{f}(s, \xi \sin \theta) \beta(|\theta|) d \theta\right| \\
& \leqq \sup _{s \geqq \frac{t_{1}}{2}} \int_{\theta=-\frac{\pi}{4}}^{\frac{\pi}{4}}|\hat{f}(s, \xi \cos \theta)-\hat{f}(s, \xi)|^{1-\left(\frac{\alpha-1}{2}+\varepsilon\right)}|\xi|^{\frac{\alpha-1}{2}+\varepsilon}|\cos \theta-1|^{\frac{\alpha-1}{2}+\varepsilon} \\
& \quad \times\left|\int_{u=0}^{1} \frac{\partial \hat{f}}{\partial \xi}(s, \xi+u \xi(\cos \theta-1)) d u\right|^{\frac{\alpha-1}{2}+\varepsilon}|\hat{f}(s, \xi \sin \theta)| \beta(|\theta|) d \theta
\end{aligned}
$$




$$
\begin{aligned}
& +\sup _{s \geqq \frac{t_{1}}{2}\left|\theta \pm \frac{\pi}{2}\right| \leqq \frac{\pi}{4}}|\hat{f}(s, \xi \cos \theta)-\hat{f}(s, \xi)||\hat{f}(s, \xi \sin \theta)| \beta(|\theta|) d \theta \\
& +\sup _{s \geqq \frac{t_{1}}{2}} \int_{\frac{3 \pi}{4} \leqq|\theta| \leqq \pi}|\hat{f}(s, \xi \cos \theta)-\hat{f}(s, \xi)||\hat{f}(s, \xi \sin \theta)| \beta(|\theta|) d \theta .
\end{aligned}
$$

We now use estimates (3.34), (3.35) and resume the computation (for all $|\xi| \geqq$ $\left.C_{3.3}, \varepsilon>0\right)$,

$$
\begin{aligned}
\sup _{s \geqq \frac{t_{1}}{2}} & |b(s, \xi)| \leqq 2 C_{2.3}(\alpha-1+2 \varepsilon)|\xi|^{\frac{\alpha-1}{2}+\varepsilon} C_{3.2}(0) \\
& \times\left(\frac{C_{3.5}\left(\varepsilon, \frac{t_{1}}{2}\right)}{1+\left|\frac{\sqrt{2}}{2} \xi\right|^{\delta-\varepsilon}}\right)^{1-\left(\frac{\alpha-1}{2}+\varepsilon\right)}\left(\frac{C_{3.7}\left(\varepsilon, t_{1}\right)}{1+\left|\frac{\sqrt{2}}{2} \xi\right|^{\delta-\varepsilon}}\right)^{\frac{\alpha-1}{2}+\varepsilon} \\
& +2 \beta_{1}\left(\frac{\pi}{4}\right)^{-\alpha} C_{3.2}(0) \frac{C_{3.5}\left(\varepsilon, \frac{t_{1}}{2}\right)}{1+\left|\frac{\sqrt{2}}{2} \xi\right|^{\delta-\varepsilon}}+2 \beta_{1}\left(\frac{3 \pi}{4}\right)^{-\alpha} C_{3.2}(0) \frac{C_{3.5}\left(\varepsilon, \frac{t_{1}}{2}\right)}{1+\left|\frac{\sqrt{2}}{2} \xi\right|^{\delta-\varepsilon}} \\
\leqq & 2 C_{2.3}(2)|\xi|^{\frac{\alpha-1}{2}-\delta+2 \varepsilon} C_{3.2}(0) 2^{\frac{\delta-\varepsilon}{2}}\left(C_{3.5}\left(\varepsilon, \frac{t_{1}}{2}\right)\right)^{1-\frac{\alpha-1}{2}+\varepsilon}\left(C_{3.7}\left(\varepsilon, t_{1}\right)\right)^{\frac{\alpha-1}{2}+\varepsilon} \\
& +4 \beta_{1}\left(\frac{\pi}{4}\right)^{-\alpha} C_{3.2}(0)|\xi|^{-\delta+\varepsilon} 2^{\frac{\delta-\varepsilon}{2}} C_{3.5}\left(\varepsilon, \frac{t_{1}}{2}\right) \\
\leqq & C_{3.8}\left(\varepsilon, t_{1}\right)|\xi|^{\frac{\alpha-1}{2}-\delta+2 \varepsilon}
\end{aligned}
$$

for some strictly positive constant $C_{3.8}\left(\varepsilon, t_{1}\right)$.

We now use estimate (3.37) to precise estimate (3.33). We get for all $t \geqq$ $t_{1},|\xi| \geqq C_{3.3}, \varepsilon>0$

$$
\begin{aligned}
|\hat{f}(t, \xi)| \leqq & C_{3.2}(0) e^{-C_{3.4} t_{1}|\xi|^{\alpha-1}}+C_{2.3}(2) C_{3.2}(0) C_{3.2}(1) \frac{t_{1}}{2}|\xi| e^{-C_{34} \frac{t_{1}}{2}|\xi|^{\alpha-1}} \\
& +\frac{C_{3.8}\left(\varepsilon, t_{1}\right)}{C_{3.4}}|\xi|^{-\frac{\alpha-1}{2} \delta+2 \varepsilon}
\end{aligned}
$$

Taking $\varepsilon=\frac{\varepsilon_{1}}{2}$, we get some strictly positive constant $C_{3.9}\left(\varepsilon_{1}, t_{1}\right)$ such that when $t \geqq t_{1},|\xi|$ is large enough,

$$
|\hat{f}(t, \xi)| \leqq C_{3.9}\left(\varepsilon_{1}, t_{1}\right)|\xi|^{-\frac{\alpha-1}{2}-\delta+\varepsilon_{1}}
$$

Finally, using estimate (3.6) for $p=0$, we obtain estimate (3.29).

We now come back to the proof of Theorem 3.1. We already know (because of estimate (3.6) when $p=0$ ) that assumption (3.28) holds when $\delta=0$. Lemma 3 clearly implies by induction that for any $\bar{t}>0, q \geqq 0$, there exists a strictly positive constant $C_{3.10}(\bar{t}, q)$ such that

$$
\forall \xi \in \mathbb{R}, \quad \sup _{t \geqq \bar{t}}|\hat{f}(t, \xi)| \leqq \frac{C_{3.10}(\bar{t}, q)}{1+|\xi|^{q}} .
$$

Using now the Sobolev inequalities (or more simply the fact that $H^{\infty}(\mathbb{R})=$ $\left.C^{\infty}(\mathbb{R})\right)$, we get Theorem 3.1 . 


\section{Regularization Properties when Some Polynomial Moments are Initially Bounded}

We extend in this section the results of Sect. 3 when assumption (3.1) does not hold any more.

Theorem 4.1. Let $f_{0} \geqq 0$ be an initial datum such that

$$
\exists r \in \mathbb{N}, r \geqq 2, C_{4.1}>0, \int_{v \in \mathbb{R}} f_{0}(v)\left(1+|v|^{2 r}+\left|\log f_{0}(v)\right|\right) d v \leqq C_{4.1},
$$

and let $\beta \geqq 0$ satisfy (2.2). Then, if $f(t, v)$ is a nonnegative solution of Eq. (1.18), (1.19), (2.2) in the sense of Eq. (2.3) with initial datum $f_{0}$, we have for all $\bar{t}>0$ and all $\varepsilon>0$ :

$$
f(t, v) \in L^{\infty}\left(\left[\bar{t},+\infty\left[t ; H^{2 r-\frac{1}{2}-\varepsilon}\left(\mathbb{R}_{v}\right)\right)\right.\right.
$$

Corollary 4.2. In particular, under the assumptions of Theorem 4.1, we have for all $\bar{t}>0$ and all $\varepsilon>0$ :

$$
f(t, v) \in L^{\infty}\left(\left[\bar{t},+\infty\left[t ; C^{2 r-2,1-\varepsilon}\left(\mathbb{R}_{v}\right)\right)\right.\right.
$$

Proof of Theorem 4.1. Corollary 4.2 is a straightforward consequence of Theorem 4.1 and of classical Sobolev inequalities (cf. [B]).

We now prove Theorem 4.1. We use the same strategy as in Theorem 3.1. Estimates (3.4) and (3.6) still hold, but only for $p \leqq 2 r$. Moreover, Eq. (3.9), (3.10), (3.12) also hold, and lead to estimate (3.27) as in Theorem 3.1.

However, Lemma 3 is changed in the following way:

Lemma 4. We make the assumptions of Theorem 4.1. We suppose moreover that there exists $\delta \geqq 0$ such that for all $t_{1}>0, \varepsilon_{1}>0$, we can find $C_{4.2}\left(\varepsilon_{1}, t_{1}\right)$ satisfying

$$
\forall \xi \in \mathbb{R}, \quad \sup _{s \geqq t_{1}}|\hat{f}(s, \xi)| \leqq \frac{C_{4.2}\left(\varepsilon_{1}, t_{1}\right)}{1+|\xi|^{\delta-\varepsilon_{1}}}
$$

Then, for all $t_{1}>0, \varepsilon_{1}>0$, we can find $C_{4.3}\left(\varepsilon_{1}, t_{1}\right)$ satisfying

$$
\forall \xi \in \mathbb{R}, \quad \sup _{s \geqq t_{1}}|\hat{f}(s, \xi)| \leqq \frac{C_{4.3}\left(\varepsilon_{1}, t_{1}\right)}{1+|\xi|^{\delta\left\{1-\frac{1}{2 r} \frac{\alpha-1}{2}\right\}+\frac{\alpha-1}{2}-\varepsilon_{1}}} .
$$

Proof of Lemma 4. We fix $t_{1}>0, \varepsilon_{1}>0$. It is clear that estimate (3.33) still holds. However, using Theorem B.2 of Appendix B, we only get for all $\varepsilon>0$ a strictly positive constant $C_{4.4}\left(\varepsilon, t_{1}\right)$ such that

$$
\forall \xi \in \mathbb{R}, \quad \sup _{s \geqq \frac{t_{1}}{2}}\left|\frac{\partial \hat{f}}{\partial \xi}(s, \xi)\right| \leqq \frac{C_{4.4}\left(\varepsilon, t_{1}\right)}{1+|\xi|^{\delta\left\{1-\frac{1}{2 r} \frac{\alpha-1}{2}\right\}+\frac{\alpha-1}{2}-\varepsilon_{1}}}
$$


Then, we note that estimate (3.36) still holds, but estimate (3.37) becomes (for all $\left.|\xi| \geqq C_{3.3}, \varepsilon>0\right)$,

$$
\begin{aligned}
& \sup _{s \geqq \frac{t_{1}}{2}}|b(s, \xi)| \leqq 2 C_{2.3}(\alpha-1+2 \varepsilon)|\xi|^{\frac{\alpha-1}{2}+\varepsilon} C_{3.2}(0) \\
& \quad \times\left(\frac{C_{4.2}\left(\varepsilon, \frac{t_{1}}{2}\right)}{1+\left|\frac{\sqrt{2}}{2} \xi\right|^{\delta-\varepsilon}}\right)^{1-\left(\frac{\alpha-1}{2}+\varepsilon\right)}\left(\frac{C_{4.4}\left(\varepsilon, \frac{t_{1}}{2}\right)}{1+\left|\frac{\sqrt{2}}{2} \xi\right|^{\delta\left(1-\frac{1}{2 r}\right)-\varepsilon}}\right)^{\frac{\alpha-1}{2}+\varepsilon} \\
& \quad+2 \beta_{1}\left(\frac{\pi}{4}\right)^{-\alpha} C_{3.2}(0) \frac{C_{4.2}\left(\varepsilon, \frac{t_{1}}{2}\right)}{1+\left|\frac{\sqrt{2}}{2} \xi\right|^{\delta-\varepsilon}}+2 \beta_{1}\left(\frac{3 \pi}{4}\right)^{-\alpha} C_{3.2}(0) \frac{C_{4.2}\left(\varepsilon, \frac{t_{1}}{2}\right)}{1+\left|\frac{\sqrt{2}}{2} \xi\right|^{\delta-\varepsilon}} \\
& \leqq \\
& \quad C_{4.5}\left(\varepsilon, t_{1}\right)|\xi|^{\frac{\alpha_{1}}{2}}+\delta\left\{-1+\frac{\alpha-1}{2} \frac{1}{2 r}\right\}+\left(2+\frac{\delta}{21}\right) \varepsilon
\end{aligned}
$$

for some strictly positive constant $C_{4.5}\left(\varepsilon, t_{1}\right)$.

Then, estimate (3.28) becomes for all $|\xi| \geqq C_{3.3}, \varepsilon>0$,

$$
\begin{aligned}
|\hat{f}(t, \xi)| \leqq & C_{3.2}(0) e^{-C_{3.4} t_{1}|\xi|^{\alpha-1}}+C_{2.3}(2) C_{3.2}(0) C_{3.2}(1) \frac{t_{1}}{2}|\xi| e^{-C_{3.4} \frac{t_{1}}{2}|\xi|^{\alpha-1}} \\
& +\frac{C_{4.5}\left(\varepsilon, t_{1}\right)}{C_{3.4}}|\xi|^{-\frac{\alpha-1}{2}-\delta\left\{1-\frac{\alpha-1}{2} \frac{1}{2 r}\right\}+\left(2+\frac{\delta}{2 r}\right) \varepsilon}
\end{aligned}
$$

Taking $\varepsilon=\varepsilon_{1}\left(2+\frac{\delta}{2 r}\right)^{-1}$, we get some strictly positive constant $C_{4.6}\left(\varepsilon, t_{1}\right)$ such that when $t \geqq t_{1},|\xi|$ is large enough,

$$
|\hat{f}(t, \xi)| \leqq C_{4.6}\left(\varepsilon, t_{1}\right)|\xi|^{-\frac{\alpha-1}{2}-\left\{1-\frac{\alpha-1}{2} \frac{1}{2 r}\right\} \delta+\varepsilon_{1}} .
$$

Then, Lemma 4 is obtained exactly as Lemma 3.

We now come back to the proof of Theorem 4.1. We already know that assumption (4.4) of Lemma 4 holds when $\delta=0$. Moreover, using Lemma 4 by induction, we can see that for all $t_{1}>0, \varepsilon_{1}>0, n \in \mathbb{N}$, there exists a strictly positive constant $C_{4.7}\left(\varepsilon_{1}, t_{1}, n\right)$ such that

$$
\forall \xi \in \mathbb{R}, \quad \sup _{t \geqq t_{1}}|\hat{f}(t, \xi)| \leqq \frac{C_{4.7}\left(\varepsilon_{1}, t_{1}, n\right)}{1+|\xi|^{\delta_{n}-\varepsilon_{1}}},
$$

where $\left(\delta_{n}\right)_{n \in \mathbb{N}}$ is the sequence defined by

$$
\begin{gathered}
\delta_{0}=0 \\
\delta_{n+1}=\delta_{n}\left\{1-\frac{\alpha-1}{2} \frac{1}{2 r}\right\}+\frac{\alpha-1}{2} .
\end{gathered}
$$

But this sequence is strictly increasing and converges to $2 r$. Therefore for all $\varepsilon>$ $0, \bar{t}>0$, there exists $C_{4.8}(\varepsilon, \bar{t})$ such that

$$
\forall \xi \in \mathbb{R}, \quad \sup _{s \geqq \bar{t}}|\hat{f}(s, \xi)| \leqq \frac{C_{4.8}(\varepsilon, \bar{t})}{1+|\xi|^{2 r-\frac{\varepsilon}{2}}} .
$$

Finally, estimate (4.13) ensures that

$$
f \in L^{\infty}\left(\left[\bar{t},+\infty\left[t ; H^{2 r-\frac{1}{2}-\varepsilon}\left(\mathbb{R}_{v}\right)\right),\right.\right.
$$

and Theorem 4.1 is proved. 


\section{Appendix A: Standard Properties of the Classical Kac Equation}

We prove in this appendix some classical facts about the spatially homogeneous Kac equation, and present some others that can easily be deduced from the theory of the Boltzmann equation (cf. [A 1]).

Theorem A.1. Let $f_{0} \geqq 0$ be an initial datum such that

$$
\int_{v \in \mathbb{R}} f_{0}(v)\left(1+|v|^{2}\right) d v<+\infty .
$$

Then, there exists a unique nonnegative solution $f(t, v)$ of Eq. (1.18), (1.19) in $L^{\infty}\left(\left[0,+\infty\left[{ }_{t}, L^{1}\left(\mathbb{R}_{v},\left(1+|v|^{2}\right) d v\right)\right)\right.\right.$ with initial datum $f_{0}$ as soon as the cross section $\beta$ in (1.19) belongs to $L^{\infty}([0, \pi])$.

This solution satisfies the conservation of mass and energy for all $t \geqq 0$ :

$$
\begin{aligned}
\int_{v \in \mathbb{R}} f(t, v) d v & =\int_{v \in \mathbb{R}} f_{0}(v) d v, \\
\int_{v \in \mathbb{R}} f(t, v)|v|^{2} d v & =\int_{v \in \mathbb{R}} f_{0}(v)|v|^{2} d v .
\end{aligned}
$$

Proof of Theorem A.1. We introduce the sequence $\left(f_{n}(t, v)\right)_{n \in \mathbb{N}}$, defined by

$$
\begin{gathered}
f_{0}(t, v)=f_{0}(v), \\
f_{n+1}(t, v)=f_{0}(v)+\int_{s=0}^{t} \int_{v_{*} \in \mathbb{R}} \int_{\theta=-\pi}^{\pi}\left\{f_{n}\left(s, v^{\prime \prime}\right) f_{n}\left(s, v_{*}^{\prime \prime}\right)\right. \\
\left.-f_{n+1}(s, v) f_{n+1}\left(s, v_{*}\right)\right\} \beta(|\theta|) d \theta d v_{*} d s,
\end{gathered}
$$

and present a proof of existence in the Cauchy-Lipschitz style.

Note that it is easy to obtain (by induction) the conservation of mass and energy for $f_{n}$ :

$$
\begin{aligned}
\int_{v \in \mathbb{R}} f_{n}(t, v) d v & =\int_{v \in \mathbb{R}} f_{0}(v) d v, \\
\int_{v \in \mathbb{R}} f_{n}(t, v)|v|^{2} d v & =\int_{v \in \mathbb{R}} f_{0}(v)|v|^{2} d v .
\end{aligned}
$$

Therefore, it is possible to write explicitly $f_{n+1}$ as a function of $f_{n}$, and the sequence (A.4), (A.5) is well defined. It is also clear that $f_{n} \geqq 0$.

Then, we define for all $n \in \mathbb{N}^{*}$,

$$
u_{n}(t)=\int_{v \in \mathbb{R}}\left|f_{n}(t, v)-f_{n-1}(t, v)\right|\left(1+|v|^{2}\right) d v .
$$

We get

$$
\begin{aligned}
u_{n+1}(t) \leqq & \int_{s=0}^{t} \int_{v \in \mathbb{R}} \int_{v_{*} \in \mathbb{R}} f_{n}\left(s, v_{*}\right)\left|f_{n}(s, v)-f_{n-1}(s, v)\right| \\
& \times\left\{\int_{\theta=-\pi}^{\pi}\left(1+|v|^{2} \cos ^{2} \theta+\left|v_{*}\right|^{2} \sin ^{2} \theta\right) \beta(|\theta|) d \theta\right\} d v d v_{*} d s \\
& +\int_{s=0}^{t} \int_{v \in \mathbb{R}} \int_{v_{*} \in \mathbb{R}} f_{n-1}(s, v)\left|f_{n}\left(s, v_{*}\right)-f_{n-1}\left(s, v_{*}\right)\right|
\end{aligned}
$$




$$
\begin{aligned}
& \times\left\{\int_{\theta=-\pi}^{\pi}\left(1+|v|^{2} \cos ^{2} \theta^{2}+\left|v_{*}\right|^{2} \sin ^{2} \theta\right) \beta(|\theta|) d \theta\right\} d v d v_{*} d s \\
& +\int_{s=0}^{t} \int_{v \in \mathbb{R}} \int_{v_{*} \in \mathbb{R}} f_{n+1}\left(s, v_{*}\right)\left|f_{n+1}(s, v)-f_{n}(s, v)\right| \\
& \times\left(1+|v|^{2}\right)\left\{\int_{\theta=-\pi}^{\pi} \beta(|\theta|) d \theta\right\} d v d v_{*} d s \\
& +\int_{s=0}^{t} \int_{v \in \mathbb{R}} \int_{v_{*} \in \mathbb{R}} f_{n}(s, v)\left|f_{n+1}\left(s, v_{*}\right)-f_{n}\left(s, v_{*}\right)\right| \\
& \quad \times\left(1+|v|^{2}\right) \int_{\theta=-\pi}^{\pi}\{\beta(|\theta|) d \theta\} d v d v_{*} d s \\
& \leqq C_{A .1} \int_{s=0}^{t}\left\{u_{n}(s)+u_{n+1}(s)\right\} d s
\end{aligned}
$$

for some strictly positive constant $C_{A .1}$. Moreover, we can prove in the same way that for all $t \geqq 0$,

$$
u_{1}(t) \leqq C_{A .2} t
$$

where $C_{A .2}>0$.

But estimate (A.9) ensures that (when $t \in[0, T], n \geqq 1$ ),

$$
u_{n+1}(t) \leqq\left(C_{A .1}+C_{A .1}^{2} T e^{C_{A .1} T}\right) \int_{s=0}^{t} u_{n}(s) d s .
$$

Therefore, for all $T>0, s \in[0, T], n \geqq 1$,

$$
u_{n}(s) \leqq\left(C_{A .1}+C_{A .1}^{2} T e^{C_{A .1} T}\right)^{n-1} \frac{s^{n}}{n !} C_{A .2} .
$$

This estimate ensures that the sequence $\left(f_{n}\right)_{n \in \mathbb{N}}$ satisfies the Cauchy property in $L_{\text {loc }}^{\infty}\left(\left[0,+\infty\left[{ }_{t}, L^{1}\left(\mathbb{R}_{v},\left(1+|v|^{2}\right) d v\right)\right)\right.\right.$. Its limit $f$ clearly satisfies Eq. (1.18), (1.19). Moreover, $f \geqq 0$ and the conservation of mass and energy $f \in L^{\infty}\left(\left[0,+\infty\left[{ }_{t}, L^{1}\left(\mathbb{R}_{v}\right.\right.\right.\right.$, $\left.\left.\left(1+|v|^{2}\right) d v\right)\right)$.

The uniqueness of such a solution is then directly obtained by a CauchyLipschitz type argument.

We now consider the polynomial moments of the solution of the spatially homogeneous Kac equation.

Theorem A.2. Let $f_{0} \geqq 0$ be an initial datum such that

$$
\int_{v \in \mathbb{R}} f_{0}(v)\left(1+|v|^{2}+\left|\log f_{0}(v)\right|\right) d v<+\infty .
$$

Then, for all $t \geqq s \geqq 0$, the unique nonnegative solution $f(t, v)$ of $E q$. (1.18), (1.19) with initial datum $f_{0}$ (when the cross section $\beta$ in (1.19) belongs to $\left.L^{\infty}([0, \pi])\right)$ satisfies

$$
\begin{aligned}
\int_{v \in \mathbb{R}} f(t, v) \log f(t, v) d v & \leqq \int_{v \in \mathbb{R}} f(s, v) \log f(s, v) d v \\
& \leqq \int_{v \in \mathbb{R}} f_{0}(v) \log f_{0}(v) d v<+\infty
\end{aligned}
$$


Moreover, if

$$
\exists r \in \mathbb{N}, \quad \int_{v \in \mathbb{R}} f_{0}(v)\left(1+|v|^{2 r}\right) d v<+\infty,
$$

there exists $C_{A .3}>0$ (independent of $\beta$ ) such that for all $t \geqq 0$ :

$$
\int_{v \in \mathbb{R}} f(t, v)\left(1+|v|^{2 r}\right) d v \leqq C_{A .3} .
$$

Proof of Theorem A.2. For estimate (A.14), we refer to [A 1], where it is proved for the Boltzmann equation (for example for Maxwellian molecules with an angular cut-off).

We now prove estimate (A.16) in the case where $r=2$. We can write

$$
\begin{aligned}
\frac{\partial}{\partial t} \int_{v \in \mathbb{R}} f(t, v)|v|^{4} d v=\int_{v \in \mathbb{R}} \int_{v_{*} \in \mathbb{R}} \int_{\theta=-\pi}^{\pi}\left(\left|v \cos \theta-v_{*} \sin \theta\right|^{4}-|v|^{4}\right) \\
\quad \times f(t, v) f\left(t, v_{*}\right) \beta(|\theta|) d \theta d v_{*} d v \\
=\int_{\theta=-\pi}^{\pi}\left\{\cos ^{4}+\sin ^{4} \theta-1\right\} \beta(|\theta|) d \theta \int_{v \in \mathbb{R}} f(t, v)|v|^{4} d v \int_{v \in \mathbb{R}} f(t, v) d v \\
\quad+6 \int_{\theta=-\pi}^{\pi} \cos ^{2} \theta \sin ^{2} \theta \beta(|\theta|) d \theta\left(\int_{v \in \mathbb{R}} f(t, v)|v|^{2} d v\right) \\
=\left\{\int_{\theta=-\pi}^{\pi} \cos ^{2} \theta \sin ^{2} \theta \beta(|\theta|) d \theta\right\}\left\{-2 \int_{v \in \mathbb{R}} f(t, v)|v|^{4} d v \int_{v \in \mathbb{R}} f(t, v) d v\right. \\
\left.\quad+6\left(\int_{v \in \mathbb{R}} f(t, v)|v|^{2} d v\right)^{2}\right\}
\end{aligned}
$$

Therefore, a simple application of the maximum principle yields

$$
\int_{v \in \mathbb{R}} f(t, v)|v|^{4} d v \leqq \sup \left(\int_{v \in \mathbb{R}} f_{0}(v)|v|^{4} d v, 3 \frac{\left(\int_{v \in \mathbb{R}} f(t, v)|v|^{2} d v\right)^{2}}{\int_{v \in \mathbb{R}}(t, v) d v}\right) .
$$

Finally, when $r>2$, the same kind of computation can be done. Note that a rigorous proof is given in the case of the Boltzmann equation with Maxwellian molecules in $[\mathrm{Tr}]$.

\section{Appendix B: Interpolation Between Derivatives}

We give here for the sake of completeness the proof of some classical results used in Sect. 2, 3 and 4.

Theorem B.1. Let $f$ lie in $C^{2}(\mathbb{R})$ and satisfy

1. There exists $C_{B .1}>0, \alpha>0$, such that

$$
\forall x \in \mathbb{R}, \quad|f(x)| \leqq \frac{C_{B .1}}{1+|x|^{\alpha}} .
$$


2. There exists $C_{B .2}>0$, such that

$$
\forall x \in \mathbb{R}, \quad\left|f^{\prime \prime}(x)\right| \leqq C_{B .2} .
$$

Then,

$$
\forall x \in \mathbb{R}, \quad\left|f^{\prime}(x)\right| \leqq \sqrt{\frac{8 C_{B .1} C_{B .2}}{1+|x|^{\alpha}}}
$$

Proof of Theorem B.1. Suppose that

$$
\left|f^{\prime}\left(x_{0}\right)\right|>\sqrt{\frac{8 C_{B .1} C_{B .2}}{1+\left|x_{0}\right|^{\alpha}}} .
$$

Then, because of estimate (B.2), for all $t \in[0,1]$,

$$
\left|f^{\prime}\left(x_{0}+t \operatorname{sgn}\left(x_{0}\right) \sqrt{\frac{2 C_{B .1}}{C_{B .2}\left(1+\left|x_{0}\right|^{\alpha}\right)}}\right)-f^{\prime}\left(x_{0}\right)\right| \leqq \sqrt{\frac{2 C_{B .1} C_{B .2}}{1+\left|x_{0}\right|^{\alpha}}} .
$$

But estimate (B.5) ensures that

$$
\left|f^{\prime}\left(x_{0}+t \operatorname{sgn}\left(x_{0}\right) \sqrt{\frac{2 C_{B .1}}{C_{B .2}\left(1+\left|x_{0}\right|^{\alpha}\right)}}\right)\right| \geqq \sqrt{\frac{2 C_{B .1} C_{B .2}}{1+\left|x_{0}\right|^{\alpha}}} .
$$

Therefore,

$$
\begin{aligned}
I & =\left|f^{\prime}\left(x_{0}+\operatorname{sgn}\left(x_{0}\right) \sqrt{\frac{2 C_{B .1}}{C_{B .2}\left(1+\left|x_{0}\right|^{\alpha}\right)}}\right)-f^{\prime}\left(x_{0}\right)\right| \\
& \geqq \frac{2 C_{B .1}}{1+\left|x_{0}\right|^{\alpha}} .
\end{aligned}
$$

But

$$
\begin{aligned}
I & \leqq\left|f\left(x_{0}+\operatorname{sgn}\left(x_{0}\right) \sqrt{\frac{2 C_{B .1}}{C_{B .2}\left(1+\left|x_{0}\right|^{\alpha}\right)}}\right)\right|+\left|f\left(x_{0}\right)\right| \\
& <\frac{2 C_{B .1}}{1+\left|x_{0}\right|^{\alpha}} .
\end{aligned}
$$

Thus, we get a contradiction and conclude that Theorem B.1 holds.

Theorem B.2. Let $p \in \mathbb{N}, p \geqq 2$, and $f$ lie in $C^{p}(\mathbb{R})$. If $f$ satisfies the following properties:

1. There exists $C_{B .3}>0, \alpha>0$, such that

$$
\forall x \in \mathbb{R}, \quad|f(x)| \leqq \frac{C_{B .3}}{1+|x|^{\alpha}} .
$$

2. There exists $C_{B .4}>0$, such that

$$
\forall q \in[1, p], \forall x \in \mathbb{R}, \quad\left|f^{(q)}(x)\right| \leqq C_{B .4} .
$$


Then, for all $\varepsilon>0$, there exists $C_{B .5}(\varepsilon)>0$, such that

$$
\forall x \in \mathbb{R}, \quad\left|f^{\prime}(x)\right| \leqq \frac{C_{B .5}(\varepsilon)}{1+|x|^{\alpha\left(1-\frac{1}{P}\right)+\varepsilon}} .
$$

Proof of Theorem B.2. We use Theorem B.1 and give a proof by induction. We know that if there exists $C_{B .6}>0$ and a finite sequence $\left(u_{q}\right)_{q \in[0, p]}$ such that for all $q \in[0, p]$,

$$
\forall x \in \mathbb{R}, \quad\left|f^{(q)}(x)\right| \leqq \frac{C_{B .6}}{1+|x|^{u_{q}}},
$$

then there exists $C_{B .7}>0$ such that for all $q \in[0, p]$,

$$
\forall x \in \mathbb{R}, \quad\left|f^{(q)}(x)\right| \leqq \frac{C_{B .7}}{1+|x|^{v_{q}}},
$$

where

$$
v_{0}=\alpha, \quad \forall i \in[1, p-1], v_{i}=\frac{1}{2}\left(u_{i-1}+u_{i+1}\right), \quad v_{p}=0 .
$$

Therefore, we define by induction the sequence

$$
r_{0}(0)=\alpha, \quad \forall i \in[1, p], r_{i}(0)=0,
$$

and

$$
r_{0}(n+1)=\alpha, \quad \forall i \in[1, p-1], r_{i}(n+1)=\frac{1}{2}\left(r_{i-1}(n)+r_{i+1}(n)\right), \quad r_{p}(n+1)=0 .
$$

It is clear that for all $n \in \mathbb{N}$, there exists $C_{B .8}(n)>0$, such that for all $q \in[0, p]$,

$$
\forall x \in \mathbb{R}, \quad\left|f^{(q)}(x)\right| \leqq \frac{C_{B .8}(n)}{1+|x|^{r_{q}(n)}} .
$$

But for all $i \in[0, p]$, the sequence $\left(r_{l}(n)\right)_{n \in \mathbb{N}}$ tends towards $r_{i}$, where

$$
r_{0}=\alpha, \quad \forall i \in[1, p-1], r_{i}=\frac{1}{2}\left(r_{i-1}+r_{i+1}\right), \quad r_{p}=0
$$

Therefore,

$$
r_{1}=\left(1-\frac{1}{p}\right) \alpha
$$

which yields Theorem B.2.

Finally, using Theorem B.2 by induction, we get the

Corollary B.3. Let $f$ lie in $C^{\infty}(\mathbb{R})$ and satisfy:

1. There exists $C_{B .9}>0, \alpha>0$, such that

$$
\forall x \in \mathbb{R}, \quad|f(x)| \leqq \frac{C_{B .9}}{1+|x|^{\alpha}} .
$$

2. For all $q \in \mathbb{N}$ there exists $C_{B .10}(q)>0$, such that

$$
\forall x \in \mathbb{R}, \quad\left|f^{(q)}(x)\right| \leqq C_{B .10}(q) .
$$


Then, for all $\varepsilon>0$, there exists $C_{B .11}(\varepsilon)>0$, such that

$$
\forall x \in \mathbb{R}, \quad\left|f^{\prime}(x)\right| \leqq \frac{C_{B .5}(\varepsilon)}{1+|x|^{\alpha-\varepsilon}} .
$$

\section{Appendix C: The Case of the Radially Symmetric 2D Boltzmann Equation with Maxwellian Molecules}

We consider now the radially symmetric solutions of the 2D spatially homogeneous Boltzmann equation with Maxwellian molecules (Note that one can prove the existence of such solutions exactly as in Sect. 2, provided of course that the initial datum is radially symmetric). The corresponding Boltzmann kernel can be written

$$
Q(f)(v)=\int_{v_{*} \in \mathbb{R}^{2}} \int_{\theta=-\pi}^{\pi}\left\{f\left(v^{\prime}\right) f\left(v_{*}^{\prime}\right)-f(v) f\left(v_{*}\right)\right\} b(|\theta|) \sin \theta d \theta d v_{*},
$$

with

$$
\begin{aligned}
& v^{\prime}=\frac{v+v_{*}}{2}+R_{0}\left(\frac{v-v_{*}}{2}\right), \\
& v_{*}^{\prime}=\frac{v+v_{*}}{2}-R_{\theta}\left(\frac{v-v_{*}}{2}\right),
\end{aligned}
$$

We suppose moreover that $b$ satisfies

$$
\sin \theta b(|\theta|) \sim K|\theta|^{-\gamma}
$$

for some $K>0$ when $\theta \rightarrow 0$ (this is the non-cut-off case) and that $b$ is regular outside 0 .

Using the fact that $f$ is radially symmetric, one can recast the kernel $Q$ under the form:

$$
\begin{gathered}
Q(f)(v)=\int_{v_{*} \in \mathbb{R}^{2}} \int_{\theta=-\pi}^{\pi}\left\{f\left(\frac{v}{2}+R_{\theta}\left(\frac{v}{2}\right)+\frac{v_{*}}{2}+R_{\theta-\pi}\left(\frac{v_{*}}{2}\right)\right)\right. \\
\left.f\left(\frac{v}{2}+R_{\theta-\pi}\left(\frac{v}{2}\right)+\frac{v_{*}}{2}+R_{\theta}\left(\frac{v_{*}}{2}\right)\right)-f(v) f\left(v_{*}\right)\right\} b(|\theta|) \sin \theta d \theta d v_{*} \\
=\int_{v_{*} \in \mathbb{R}^{2}} \int_{\theta=-\pi}^{\pi}\left\{f\left(R_{\frac{\theta}{2}}(v) \cos \left(\frac{\theta}{2}\right)+R_{\frac{\theta}{2}-\frac{\pi}{2}}\left(v_{*}\right) \sin \left(\frac{\theta}{2}\right)\right)\right. \\
\left.f\left(R_{\frac{\theta}{2}-\frac{\pi}{2}}(v) \sin \left(\frac{\theta}{2}\right)+R_{\frac{\theta}{2}}\left(v_{*}\right) \cos \left(\frac{\theta}{2}\right)\right)-f(v) f\left(v_{*}\right)\right\} b(|\theta|) \sin \theta d \theta d v_{*} \\
=\int_{v_{*} \in \mathbb{R}^{2}} \int_{\theta=-\pi}^{\pi}\left\{f\left(v \cos \left(\frac{\theta}{2}\right)-v_{*} \sin \left(\frac{\theta}{2}\right)\right) f\left(v \sin \left(\frac{\theta}{2}\right)+v_{*} \cos \left(\frac{\theta}{2}\right)\right)\right. \\
\left.-f(v) f\left(v_{*}\right)\right\} b(|\theta|) \sin \theta d \theta d v_{*} .
\end{gathered}
$$


Thus, we can see that the equation is very similar to the Kac equation. The main difference is simply that now $v$ is in $\mathbb{R}^{2}$ instead of $\mathbb{R}$. It is then possible to prove all the theorems of the previous sections with exactly the same proof.

Note however that for the 3D radially symmetric solutions of the spatially homogeneous Boltzmann equation with Maxwellian molecules, the analogy with the Kac equation is not so clear. This case shall be discussed in a future work.

Acknowledgement. I would like to thank Professor Golse for his valuable remark during the preparation of this work.

\section{References}

[A 1] Arkeryd, L.: On the Boltzmann equation, I and II. Arch. Rat. Mech. Anal. 45, 1-34 (1972)

[A 2] Arkeryd, L.: Intermolecular forces of infinite range and the Boltzmann equation. Arch. Rat. Mech. Anal. 77, 11-21 (1981)

[A 3] Arkeryd, L.: Stability in $L^{1}$ for the spatially homogeneous Boltzmann equation. Arch. Rat. Mech. Anal. 103, 151-167 (1988)

[B] Brezis, H.: Analyse fonctionnelle, théorie et applications. Paris: Masson, (1983)

[Ce] Cercignani, C.: The Boltzmann equation and its applications. Berlin, Heidelberg, New York: Springer, (1988)

[Ch, Co] Chapman, S., Cowling, T.G.: The mathematical theory of non-uniform gases. London: Cambridge Univ. Press., (1952)

[Dg, Lu] Degond, P., Lucquin-Desreux, B.: The Fokker-Planck asymptotics of the Boltzmann collision operator in the Coulomb case. Math. Mod. Meth. Appl. Sc. 2 (1992)

[De 1] Desvillettes, L.: Some applications of the method of moments for the homogeneous Boltzmann and Kac equations. Arch. Rat. Mech. Anal. 123, 387-404 (1993)

[De 2] Desvillettes, L.: On asymptotics of the Boltzmann equation when the collisions become grazing. Tr. Th. and Stat. Phys. 21, 259-276 (1992)

[De 3] Desvillettes, L.: Convergence to equilibrium in large time for Boltzmann and B.G.K equations. Arch. Rat. Mech. Anal. 110, 73-91 (1990)

[DP, L] DiPerna, R.J., Lions, P-L.: On the Cauchy problem for Boltzmann equations, Global existence and weak stability. Ann. Math. 130, 321-366 (1989)

[El] Elmroth, T.: Global boundedness of moments of solutions of the Boltzmann equation for forces of infinite range. Arch. Rat. Mech. Anal. 82, 1-12 (1983)

[G] Gabetta, E.: On a conjecture of McKean with application to Kac's model. To appear in Tr. Th. and Stat. Phys.

[Gr] Grad, H.: Principles of the kinetic theory of gases. Flügge's Handbuch der Physik. 12, 205-294 (1958)

[K] Kac, M.: Probability and related topics in the Physical Sciences. NewYork: (needs publisher) (1959)

[L 1] Lions, P-L.: Compactness in Boltzmann's equation via Fourier integral operators and applications, I, II and III. Preprint

[L 2] Lions, P-L.: On Boltzmann and Landau equations. Cahiers de mathématiques de la décision, n. 9220, and to appear in Phil. Trans. Roy. Soc.

[MK] McKean, H.P.: Speed of approach to equilibrium for Kac's caricature of a Maxwellian gas. Arch. Rat. Mech. Anal. 21, 347-367 (1966)

[Tr] Truesdell, C.: On the pressures and the flux of energy in a gas according to Maxwell's kinetic theory II. J. Rat. Mech. Anal. 5, 55 (1955)

[Tr, Mu] Truesdell, C., Muncaster, R.: Fundamentals of Maxwell kinetic theory of a simple monoatomic gas. New York: Acad. Press., (1980)

[We] Wennberg, B.: Preprint 